INPLASY

PROTOCOL

To cite: Zhang et al. Traditional Chinese medicine for cancerrelated fatigue: a systematic review and meta-analysis. Inplasy protocol 202180114. doi:

10.37766/inplasy2021.8.0114

Received: 30 August 2021

Published: 30 August 2021

Corresponding author:

Chi Zhang

940399721@qq.com

Author Affiliation:

Guangzhou university of

chinese medicine.

Support: Not applicable.

Review Stage at time of this submission: The review has not yet started.

Conflicts of interest:

None declared.

\section{Traditional Chinese medicine for cancer-related fatigue: a systematic review and meta-analysis}

\author{
Zhang, C1; Guo, W22.
}

Review question / Objective: To investigate the efficacy of Traditonal Chinese medicine in treatment of CRF.

Condition being studied: The treatment of CRF by traditional Chinese medicine is controversial. We conducted a metaanalysis and systematic review to evaluate the effect, with a view to providing some guidance for clinical application. All research members have mastered meta-analysis techniques, can use databases to screen documents, and use software for statistical analysis.

Information sources: We will start electronic searches from PubMed, MEDLINE, Embase, Web of Science,Cochrane Central Register of Controlled Trials (Central), China National Knowledge Infrastructure (CNKI), China Biomedical Literature Database (CBM), China Science Journal Database (VIP) and Wanfang Database. The search date is from the database to July 30, 2021, and the search language is limited to Chinese and English. In addition, we will manually retrieve other resources, including reference lists of identified publications, conference articles and grey literature.

INPLASY registration number: This protocol was registered with the International Platform of Registered Systematic Review and Meta-Analysis Protocols (INPLASY) on 30 August 2021 and was last updated on 30 August 2021 (registration number INPLASY202180114).

\section{INTRODUCTION}

Review question / Objective: To investigate the efficacy of Traditonal Chinese medicine in treatment of CRF.
Condition being studied: The treatment of CRF by traditional Chinese medicine is controversial. We conducted a metaanalysis and systematic review to evaluate the effect, with a view to providing some guidance for clinical application. All 
research members have mastered metaanalysis techniques, can use databases to screen documents, and use software for statistical analysis.

\section{METHODS}

Search strategy: English database search: )(()(广()(((randomized controlled trial[Title) Abstract]) OR controlled clinical trial[Title/ Abstract]) OR randomized[Title/Abstract]) OR placebo[Title/Abstract]) OR clinical trials as topic[Title/Abstract]) OR randomly[Title/Abstract]) OR trial[Title/ Abstract])) OR "Randomized Controlled Trial" [Publication Type])) AND (("Medicine, Chinese Traditional"[Mesh]) OR ()(()((()Traditional Chinese Medicine[Title/ Abstract]) OR Chung I Hsueh[Title/ Abstract]) OR Zhong Yi Xue[Title/Abstract]) OR Chinese Traditional Medicine[Title/ Abstract]) OR Drugs, Chinese Herbal[Title/ Abstract]) OR Chinese Drugs, Plant[Title/ Abstract]) OR Chinese Herbal Drugs[Title/ Abstract]) OR Plant Extracts, Chinese[Title/ Abstract]) OR Decoction[Title/Abstract]) OR Prescription[Title/Abstract]))) AND

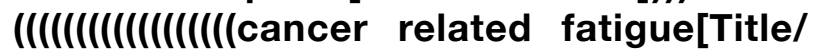
Abstract]) OR CRF[Title/Abstract]) OR chemotherapy related fatigue[Title/ Abstract]) OR treatment related fatigue[Title/Abstract]) OR apathy[Title/ Abstract]) OR apathetic[Title/Abstract]) OR I assitude [ Title / Abstract]) OR weakness [Title/Abstract]) OR lethargy[Title/Abstract]) OR lethargic[Title/ Abstract]) OR tired[Title/Abstract]) OR weary[Title/Abstract]) OR weariness[Title/ Abstract]) OR exhaustion[Title/Abstract]) OR exhausted[Title/Abstract]) OR Iackluster[Title/Abstract])) OR "Fatigue"[Mesh])) AND ()((()(广(()((Neoplasias [Title/Abstract]) OR Neoplasm[Title/ Abstract]) OR Tumors[Title/Abstract]) OR Tumor[Title/Abstract]) OR Cancer[Title/ Abstract]) OR Cancers[Title/Abstract]) OR Malignancy[Title/Abstract]) OR Malignancies[Title/Abstract]) OR Malignant Neoplasms[Title/Abstract]) OR Malignant Neoplasm[Title/Abstract]) OR Neoplasm, Malignant[Title/Abstract]) OR Neoplasms, Malignant[Title/Abstract])) OR "Neoplasms"[Mesh]).
Participant or population: The research object is patients diagnosed as malignant tumor, accompanied by cancer-related fatigue after pathological and (or) cytological examination. The diagnostic criteria of cancer were based on the FAB classification diagnostic criteria which were determined by France, America, and British, FAB collaborative group, or World Health Organization (WHO) classification. The diagnostic criteria for cancer-related fatigue is based on International Classification of Diseases Tenth Edition (ICD-10). All cancer types were included, because we assumed that CRF is a general cancer problem and that the working mechanisms and effects of the different interventions targeting CRF would be similar across all cancer diagnoses.

Intervention: The experimental group is treated with TCM treatment, including decoctions, proprietary Chinese medicines, injections, etc., which can be treated alone or in combination with conventional treatments.

Comparator: The control group is treated with conventional modern therapy, such as radiotherapy, chemotherapy, supportive treatment, health education, placebo, etc., excluding TCM treatment.

Study designs to be included: Randomized controlled trials (RCTs) and quasi-RCTs (qRCTs) evaluated TCM for treating CRF.

Eligibility criteria: Pharmacological research, pharmacokinetic research, animal experiment, nursing experience, adverse reaction report, case reports, experience summaries, theoretical discussions, reviews, abstracts, pilot studies, protocol, RCTs using TCM in the control group or reporting incomplete data, Duplicate documents.

Information sources: We will start electronic searches from PubMed, MEDLINE, Embase, Web of Science, Cochrane Central Register of Controlled Trials (Central), China National Knowledge Infrastructure (CNKI), China Biomedical Literature Database (CBM), China Science 
Journal Database (VIP) and Wanfang Database. The search date is from the database to July 30,2021 , and the search language is limited to Chinese and English. In addition, we will manually retrieve other resources, including reference lists of identified publications, conference articles and grey literature.

Main outcome(s): The primary outcome is the severity of fatigue assessed using total effective rate and two validated tools (Piper Fatigue Scale and Karnofsky performance status), result of total effective rate is produced by calculating the ratio of the number of people who are significantly effective and stable in improving fatigue symptoms after treatment to the total sample. The secondary outcomes are the 'expression level of HGB, IL-2, CD4+ T cell, CD8+ $T$ cell and NK cell in serum of participants.

Quality assessment / Risk of bias analysis: This study was independently searched by two researchers (ZC and GW). They independently screened titles and abstracts for eligibility and read full texts independently to extract data. In case of disagreement, consensus was reached through discussion. Modified Jadad scale (total score is seven, higher score is better quality) was used to estimate the quality of the included RCTs, of which one to three were classified as low-quality, and four to seven were classified as high-quality.

Strategy of data synthesis: RevMan5.0 software was used to analyze the data and compare the difference between the experimental group and the control group, for the efficacy of TCM in treating cancerinduced fatigue. If the meta-analysis is lowlevel heterogeneity (1250\%), we will use the random-effect model with $95 \% \mathrm{Cl}$ to analyze. Dichotomous data use relative risk ratio (RR) and risk difference (RD) reports. Because the scale of the research we chose is the identical, so successive data will be reported by using mean difference (MD), Sensitivity analysis was carried out by changing the combined effect model, subgroup analysis, and eliminating study one by one.

Subgroup analysis: If there are obvious heterogeneity, we will conduct subgroup analysis to identify the sources of heterogeneity. We will conduct subgroup analysis according $t \mathrm{o}$ different course time or different outcome indicators.

Sensitivity analysis: When sufficient trials are available, sensitivity analysis will be performed by sequentially eliding each trial to check the robustness of the final results.

Country(ies) involved: China.

Keywords: Fatigue; Cancer; Meta-analysis; Traditional Chinese medicine.

Contributions of each author:

Author 1 - Chi zhang - Manuscript writing, data collection and analysis.

Email: 940399721@qq.com

Author 2 - Wei Guo - Data collection and analysis.

Email: ydksbs@163.com 